\title{
Competencia entre tres especies de Nothofagus y Pseudotsuga menziesii en plantaciones mixtas jóvenes, establecidas en la precordillera andina de Valdivia
}

Competition between three species of Nothofagus and Pseudotsuga menziesii in young mixed stands planted in the foothill zone of the Andes Mountains, Valdivia/Chile

\author{
MARION WIENSTROER ${ }^{1}$, HERBERT SIEBERT $^{2}$, BURKHARD MÜLLER-USING $^{3}$ \\ ${ }^{1}$ Landesforstverwaltung Nordrhein-Westfalen, Düsseldorf, Alemania. \\ ${ }^{2}$ Instituto de Silvicultura, Universidad Austral de Chile, hsiebert49@ hotmail.com \\ ${ }^{3}$ Instituto de Silvicultura, Universidad Austral de Chile, burkhardmusing@yahoo.de
}

\section{SUMMARY}

Forest plantations composed of four species, three from the genus Nothofagus and one of Pseudotsuga menziesii, were evaluated in the X Region of Chile, close to the Maihue Lake. Plantations established in the open and in gaps of no more than 0.04 ha were included in this study. Under both conditions, plots were established on sun exposed slopes (north to west exposures) and shady slopes (east to south exposures), with care taken to make sufficient repetitions. The natural age stages of the plantations were pole stands in the open and thickets in the gaps. The objective of this study was to analyze the development of the young mixed stands of Roble (Nothofagus obliqua), Raulí (N. alpina), Coigüe (N. dombeyi) and Douglas Fir (Pseudotsuga menziesii). This was especially with regard to growth parameters and stem quality, comparing the influence of exposure as well as the plantation in the open and in gaps. Answers as to when and how to undertake the first thinning were expected. Diameter at breast height (DBH) and tree heights of a representative sample were firstly taken and means calculated to make comparisons on a species level. This also took into account the exposure and whether the plantations had been established in the open or in gaps. The second step consisted of studying the competition between trees on an individual scale. In order to realize this objective, 270 trees were selected across all plots and classified with regard to their relative position in comparison to their four closest neighbors. Stem quality was also evaluated. To minimize subjectivity in this process, a system of five quality criteria were developed. In the open, the development of all four species was superior to that in the gaps. However, stem quality was higher in the gaps. In the open, at an age of 10 years, the competitive power of Roble, Raulí and Coigüe was superior to that of the Douglas Fir, whereas in the gaps Douglas Fir, together with Coiguie, attained tree heights superior to that of Roble and Raulí. A study of the competitive status of trees in comparison to their neighbors was included in this study and provides figures of dominant and codominant trees of both good and bad stem forms under all growth conditions. From these figures, recommendations were made about when and how to manage the first tree thinning operation.

Key words: mixed plantations, interspecific competition, Nothofagus genus, Douglas Fir, precommercial thinning.

\section{RESUMEN}

En la X Región de Chile, al norte del Lago Maihue, se evaluaron plantaciones mixtas de tres especies del género Nothofagus y Pseudotsuga menziesii realizadas por una parte en campo abierto y por la otra en 14 claros de 0,04 ha en promedio. En ambas condiciones se establecieron parcelas en laderas asoleadas (exposiciones Norte hasta Oeste) y laderas sombrías (exposiciones Este hasta Sur) procurando repeticiones. Las fases de desarrollo natural eran latizales en el campo abierto y brinzales en los claros. El objetivo de este estudio fue investigar el estado de desarrollo de plantaciones mixtas de roble (Nothofagus obliqua), raulí (Nothofagus alpina), coigüe (Nothofagus dombeyi) y pino oregón (Pseudotsuga menziesii), especialmente en relación a crecimiento y calidad, y en función de los factores de exposición y sombra lateral (claros). De este análisis se esperaron indicaciones para la primera intervención silvícola, tanto con respecto al tiempo oportuno de su realización como en relación al tipo y número 
BOSQUE 24(3): 17-30, 2003

Competencia entre tres especies de Nothofagus y Pseudotsuga menziesii en plantaciones mixtas jóvenes...

de los árboles a eliminar. En un primer estudio se midieron diámetros a la altura de pecho (DAP) y alturas de un colectivo representativo, calculando promedios para hacer comparaciones a nivel de especies, exposiciones y entre la situación en campo abierto y en los claros. Un segundo estudio analizó la situación de competencia a escala individual. Para ello fueron seleccionados 270 individuos a través de todas las parcelas, los que fueron clasificados según su posición relativa frente a sus cuatro vecinos más cercanos y además evaluados según la calidad de sus fustes. Para minimizar la subjetividad de esta última estimación se elaboró un esquema con cinco criterios cualitativos. En campo abierto el desarrollo de todas las especies fue más acelerado que en los claros, pero la calidad de los fustes fue generalmente mejor en los claros. Mientras que en campo abierto, a la edad de diez años, roble, raulí y coigüe se mostraron más competitivos que pino oregón, no fue así en los claros, donde pino oregón y coigüe tenían mayores alturas que roble y raulí. El estudio de competencia y calidad a escala individual arrojó números de árboles dominantes y codominantes con buena y mala forma en todas las condiciones estudiadas. De estas cifras se derivaron pautas para el primer raleo en las plantaciones en campo abierto.

Palabras claves: plantaciones mixtas, competencia interespecífica, especies Nothofagus, pino oregón, raleo precomercial.

\section{INTRODUCCION}

En la discusión sobre la futura imagen de los bosques en Chile prevalece todavía el concepto de mantener por separado las masas de los bosques nativos y las plantaciones formadas por especies exóticas de rápido crecimiento. Este concepto ha traído consigo una efervescente polémica sobre la sustitución del bosque nativo por plantaciones del tipo antes indicado, polémica que ha llevado a un cierto estancamiento en la silvicultura del país.

Sin embargo, a criterio de algunos expertos silvícolas en Europa (Otto 1987) y Chile (Siebert 1999) la polarización entre las dos doctrinas se puede eliminar con la idea de integrar especies exóticas, después de pruebas de aptitud, en la creación de los futuros bosques, mezclándolos con especies nativas. Sin embargo, en esta materia todavía son escasos los conocimientos sobre el comportamiento competitivo de las especies constitutivas de tales mezclas, lo que también es el caso para rodales mixtos entre especies de Nothofagus y Pseudotsuga menziesii en distintas condiciones de sitio en Chile.

El presente trabajo es una contribución a esta temática, aunque no pueda presentar más que un enfoque, limitado en tiempo y espacio, al problema de la compatibilidad entre especies forestales nativas y exóticas en Chile. El objetivo principal es investigar el estado de desarrollo de plantaciones mixtas de roble (Nothofagus obliqua Mirb. BL), raulí (Nothofagus alpina Poepp. et Endl.), coigüe (Nothofagus dombeyi Mirb. Oerst.) y pino oregón (Pseudotsuga menziesii Mirb. Franco), especialmente en relación a crecimiento y calidad y en función de los factores de exposición y sombra lateral.

Para fines de manejo de rodales es insuficiente estimar las posibilidades que tenga una especie frente a las demás con las que se encuentra en mezcla sólo mediante valores promedios de altura. Es más interesante saber en cuántos casos la especie en consideración predomina o codomina en la mezcla para pronosticar su futura participación en el rodal mixto. Como en el rodal maduro se necesitan menos árboles para ocupar el espacio que en las etapas anteriores, basta con una cantidad de, por ejemplo, 75 árboles dominantes y de buena calidad por hectárea para garantizarle a esta especie, mediante algunas cortas sucesivas de liberación de copas, una participación de un 50\% en el rodal maduro.

En este sentido un estudio de competencia $a$ escala individual arrojaría los números de árboles que existen en un momento dado, con buenas características de competitividad y al mismo tiempo de calidad fustal satisfactoria como para seguir trabajando con ellos.

Es por ello que fuera de una comparación de valores promedios en altura y diámetro se emprendió este estudio de competencia a escala individual.

\section{MATERIAL Y METODOS}

Area de estudio y diseño experimental: Se buscaron plantaciones mixtas de las especies mencionadas, cuya edad fuera tan avanzada que hubieran entrado en competencia interespecífica, poblando 
tanto pendientes sombrías como asoleadas y plantadas por un lado en sitios abiertos y por el otro en claros que contaran con un régimen de sombra lateral, proveniente de las masas adyacentes de rodales más viejos.

Se encontró este tipo de plantaciones en extensión suficiente y aparente homogeneidad entre sí en el Fundo Arquilhue, situado en la X Región chilena, a los $42^{\circ}$ Latitud Sur y $71^{\circ}$ Longitud Oeste. Estas plantaciones se crearon desde el año 1991 en adelante. Cabe señalar que con la técnica de plantar en claros se ha empezado un año más tarde en este fundo, de manera que las parcelas escogidas con sombra lateral tienen 9 años de edad y las plantaciones de campo abierto 10.

Bajo el término de "ladera sombría" se entienden las pendientes expuestas desde el Sur, Sureste hasta el Este, mientras que las laderas asoleadas son los que dan al Norte, Noroeste y Oeste. Este concepto se basa en las particularidades meteorológicas que se encuentran en Chile alrededor de $\operatorname{los} 40^{\circ}$ de Latitud Sur, donde el promedio de horas con sol es generalmente más corto en las mañanas, es decir, con la posición Este del Sol, y bastante más largo en las tardes, es decir, con la posición Oeste del sol. Este efecto es aún más pronunciado en la precordillera y cordillera que en el valle central, y se suma a este fenómeno el efecto de la sombra orográfica generado por la cordillera de los Andes al levantarse el sol en la mañana.

Las parcelas de medición en las plantaciones realizadas en campo abierto fueron localizadas de tal forma que dos de ellas tenían exposiciones más cálidas $(\mathrm{N}, \mathrm{NOE}, \mathrm{OE})$ y dos de sombrío (, $\mathrm{SE}$, E), incluyendo cada una de ellas un mínimo de 30 árboles de las cuatro especies en estudio. Las características de esta parte del ensayo se presentan en el cuadro 1 .

En base a este diseño se midieron y evaluaron 120 individuos por especie en el programa de medición y evaluación cualitativa, lo que suma 480 objetos de medición de todas las especies en conjunto.

El arreglo de los individuos por especie en cada una de las plantaciones se muestra en la figura 1 .

Se trataba de un espaciamento cuadrático en que las plantas tenían una distancia de 2,5 m entre sí. Como una particularidad inalterable se debe mencionar que con este diseño la especie Roble tenía como vecinos directos a un coigüe o pino oregón, pero nunca a un raulí. El coigüe, a su vez, contaba con los vecinos directos de raulí y roble, pero nunca con pino oregón, etc. Este hecho trajo consigo una cierta limitación en la comparación del poder competitivo que tiene cada especie frente a las demás.

Para estudiar el comportamiento de las especies bajo condiciones de sombra lateral se buscaron claros en un extenso renoval de roble, adyacente a las plantaciones anteriormente mencionadas, en las que las mismas especies habían sido plantadas. En total se encontraron 8 claros en laderas sombrías y 6 en laderas asoleadas (cuadro 2).

La superficie de los claros se estimó a través del promedio de dos diámetros, y usando la fórmula $\mathrm{D}^{2} \pi / 4$, donde $\mathrm{D}$ significa diámetro a la altura del pecho, definiendo de manera simplificada la forma de la superficie de cada claro como circular. Con respecto al tamaño máximo de los claros a elegir se puso un límite de alrededor de 0,05 ha.

\section{CUADRO 1}

Características de las parcelas en campo abierto. Information about the plots established in the open.

\begin{tabular}{|l|c|c|c|c|r|}
\hline Campo abierto & Sector & Inclin. mínima & Inclin. máxima & Inclin. media & Azimut \\
\hline Ladera asoleada 1 & Las Quemas & $11^{\circ}$ & $17,5^{\circ}$ & $14,5^{\circ}$ & $5^{\circ}$ \\
Ladera asoleada 2 & Chollelhue & $12,5^{\circ}$ & $19^{\circ}$ & $14,7^{\circ}$ & $260^{\circ}$ \\
\hline Ladera de sombrío 1 & Las Quemas & $11^{\circ}$ & $16^{\circ}$ & $12,7^{\circ}$ & $164^{\circ}$ \\
Ladera de sombrío 2 & Las Quemas & $10^{\circ}$ & $15,5^{\circ}$ & $12,8^{\circ}$ & $155^{\circ}$ \\
\hline
\end{tabular}


BOSQUE 24(3): 17-30, 2003

Competencia entre tres especies de Nothofagus y Pseudotsuga menziesii en plantaciones mixtas jóvenes...

\begin{tabular}{|l|l|l|l|}
\hline Ro & Co & Ro & Co \\
\hline P.O & Ra & P.O & Ra \\
Ro & Co & Ro & Co \\
P.O & Ra & P.O & Ra \\
\hline
\end{tabular}

Figura 1. Esquema de plantación en las parcelas: Ro $=$ Roble, $\mathrm{Ra}=$ Raulí, $\mathrm{Co}=$ Coigüe, $\mathrm{P} . \mathrm{O}=$ Pino Oregón.

Plant spacing in the plots: Ro = Roble, Ra $=$ Raulí, $\mathrm{Co}=$ Coigüe and P.O = Douglas Fir.

Los claros tenían el mismo esquema de plantación que en campo abierto. En un primer paso se identificaron las plantas a medir dejando de lado la regeneración de roble que también estaba presente. Para representar los diferentes niveles de luz dentro de cada claro se trazó un gradiente desde el borde septentrional, es decir, del lado más afectado por la sombra lateral hacia el centro del claro. En este gradiente se midieron 5 plantas por especie. Con este procedimiento se garantizó que se evaluasen colectivos comparables de plantas que además fuesen característicos para el claro al que pertenecían.

Análisis cuantitativo: Las alturas de los 480 árboles en campo abierto y de las 280 plantas en los claros se midieron usando una vara telescópica; los diámetros a la altura de pecho (DAP) con forcípula. La estimación de superioridad, igualdad o inferioridad en altura de los árboles centrales con respecto a sus 4 vecinos se realizó en forma ocular. Se consideró árbol superior o inferior aquel árbol cuya diferencia en altura era por lo menos de 1,5 m respecto a su vecino. Para este procedimiento se usó el mismo colectivo de árboles que más tarde se escogieron para la evaluación cualitativa, es decir, 50 individuos por especie y parcela.

Análisis cualitativo: La evaluación cualitativa se hizo con un esquema que abarca 5 criterios, a saber, presencia de daños, presencia y posición de bifurcaciones, rectitud y excentricidad del fuste, tal como el grosor de las ramas. Estos parámetros se calificaron en base de las particularidades que

CUADRO 2

Características de los claros inventariados.

Information about the gaps selected to be measured.

\begin{tabular}{|c|c|c|c|c|}
\hline $\begin{array}{c}\text { Exposición } \\
\text { de los claros }\end{array}$ & Sector & $\begin{array}{c}\text { Inclinación } \\
(\%)\end{array}$ & Azimut & $\begin{array}{c}\text { Area del claro } \\
\text { (ha) }\end{array}$ \\
\hline sombrío 1 & Las Quemas & 31 & 230 & 0,0443 \\
sombrío 2 & Las Quemas & 15 & 200 & 0,0314 \\
sombrío 3 & Las Quemas & 18 & 155 & 0,0241 \\
sombrío 4 & Las Quemas & 15 & 145 & 0,0207 \\
sombrío 5 & Las Quemas & 17 & 130 & 0,0177 \\
sombrío 6 & Las Quemas & 27 & 240 & 0,0491 \\
sombrío 7 & Las Quemas & 26 & 210 & 0,0241 \\
sombrío 8 & Las Quemas & 22 & 150 & 0,0314 \\
\hline asoleado 1 & Las Canoas & 17 & 345 & 0,0276 \\
asoleado 2 & Las Canoas & 18 & 340 & 0,0355 \\
asoleado 3 & Las Canoas & 8 & 260 & 0,0443 \\
asoleado 4 & Las Canoas & 28 & 290 & 0,0314 \\
asoleado 5 & Las Canoas & 14 & 310 & 0,0541 \\
asoleado 6 & Las Canoas & 8 & 310 & 0,0594 \\
\hline
\end{tabular}


tiene la especie como tal. Para ello se organizó un entrenamiento de tres días, en el cual el personal se familiarizó también con el sistema numérico empleado para la calificación de cada uno de los criterios. Finalmente se agregaron las "notas" dadas en cada criterio a una calificación general, la que desembocó en la determinación de los “árboles del futuro". El cuadro 3 muestra el desglose numérico de los cinco criterios con que se hizo la evaluación.

Análisis estadístico: La comparación en DAP y altura entre especies y exposiciones se realizó a través del análisis de varianza. Una condición para aplicar este método estadístico reside en que los datos sean seleccionados de la población en forma aleatoria e independiente, requisito que no se podia cumplir en el presente muestreo, como es usual en terreno de muy dificil accesibilidad. Sin embargo, en tales condiciones un test de permutación puede generar una distribución de valores observados de F; esta se compara con la distribución teórica de los valores $\mathrm{F}$ y al mostrarse una similitud suficiente se permite el uso del análisis de varianza (Yandell 1997). Así se procedió en el caso de este trabajo; como análisis posterior se usó el test de Scheffé (Sachs 1984).

Para la presentación de los datos sobre calidad en escala ordinal se presentan tablas de contingencia multidimensionales. Es con un análisis loglineal que se comprueba o rechaza la relación entre los variables (por ejemplo: tendencia a bifurcación con especie, exposición con daño fustal, etc.) y con eso se deducen conclusiones con respecto a la optimización de técnicas silviculturales.

El procedimiento para definir la superioridad o inferioridad relativa en la competencia entre especies no genera condiciones en que se pueden apli-

\section{CUADRO 3}

Esquema de evaluación de los árboles de destacada posición relativa.

Quality components used for the evaluation of trees with a dominant or codominant position.

\begin{tabular}{|c|c|c|c|}
\hline \multicolumn{2}{|c|}{ Daño } & \multicolumn{2}{|c|}{ Bifurcaciones } \\
\hline 1 & Sin daño & 1 & Ninguna bifurcación 1 \\
\hline 2 & Con daño & 2 & Bifurcación arriba de $9 \mathrm{~m}$ \\
\hline 3 & Bifurcación entre 6 y 9 m & & \\
\hline 4 & Bifurcación abajo de $6 \mathrm{~m}$ & & \\
\hline \multicolumn{2}{|c|}{ Rectitud del fuste } & \multicolumn{2}{|c|}{ Corte transversal del fuste } \\
\hline 1 & Recto & 1 & Forma circular \\
\hline 2 & Ligeramente curvado & 2 & Ligeramente elíptico \\
\hline 3 & Curvado en una dimensión & 3 & Medianamente elíptico \\
\hline 4 & Curvado en varias dimensiones & 4 & Elíptico \\
\hline 5 & Fuertemente curvado y/o torcido & 5 & Fuertemente elíptico \\
\hline \multicolumn{2}{|c|}{ Grosor de las ramas } & & \\
\hline 1 & Ramas muy finas & & \\
\hline 2 & Ramas finas & & \\
\hline 3 & Ramas regulares & & \\
\hline 4 & Ramas moderadamente gruesas & & \\
\hline 5 & Ramas gruesas & & \\
\hline 6 & Ramas gruesas hasta muy gruesas & & \\
\hline 7 & Ramas muy gruesas & & \\
\hline
\end{tabular}


BOSQUE 24(3): 17-30, 2003

Competencia entre tres especies de Nothofagus y Pseudotsuga menziesii en plantaciones mixtas jóvenes...

car tests paramétricos, por lo cual se hizo uso del test de Kolmogoroff-Smirnoff (Sachs 1984) para comprobar o rechazar la homogeneidad de dos muestras (por ejemplo: si el desarrollo en altura de Raulí es significativamente $>$ o $<$ que el de coigüe o no).

\section{RESULTADOS}

Parámetros de crecimiento en las plantaciones establecidas en campo abierto: Los DAP oscilan entre 7,4 y 10,9 cm después de 10 años de desarrollo, mostrando las especies roble y coigüe ventaja sobre raulí y pino oregón (cuadro 4). El análisis de varianza arrojó diferencias según especies y según exposición. El test de Scheffé mostró que hubo diferencia significativa en el crecimiento diamétrico entre las especies roble y raulí, así como entre roble y pino oregón. También se distinguió coigüe de las especies raulí y pino oregón. Por otro lado, no son significativamente distintas las diferencias entre roble y coigüe tal como entre raulí y pino oregón.

La influencia de la exposición sobre el desarrollo diamétrico es altamente significativa sólo en el caso del raulí de acuerdo al test de Scheffé. Las demás especies no mostraron reacción signi- ficativa por su desarrollo diamétrico al factor exposición.

Las alturas oscilan entre 6,04 y 9,10 m (cuadro 5). La especie roble tiene un desarrollo significativamente mayor que las otras especies en exposición Norte, mientras que en pendiente Sur el raulí supera al roble. El pino oregón, a su vez, figura como la especie de peor desarrollo en altura en ambas exposiciones. El análisis de varianza arrojó una influencia altamente significativa del factor especie. Sólo entre roble y raulí eran insignificantes las diferencias, compensándose ventajas del raulí sobre el roble en la exposición Sur con el caso opuesto en exposición Norte. Todas las demás diferencias entre las especies son significativas.

La influencia de la exposición no fue significativa para roble, coigüe y pino oregón, pero sí para raulí, esta especie tuvo un desarrollo altitudinal mucho mejor en la pendiente Sur que en la Norte.

Parámetros de crecimiento en los claros: En la elaboración de los datos se juntaron los 8 claros en laderas asoleadas, realizándose el mismo procedimiento para los 6 en laderas sombrías.

El desarrollo en diámetro de roble y raulí fue significativamente inferior que el de coigüe y pino oregón en ambiente de sombra lateral (figura 2).

\section{CUADRO 4}

Promedio de los DAP y sus respectivos errores $(\mathrm{cm})$ por especie en las plantaciones mixtas. Mean values of $\mathrm{DBH}$ and their errors $(\mathrm{cm})$ for each tree specie in the mixed plantations.

\begin{tabular}{|c|c|c|c|c|c|c|}
\hline & & \multicolumn{5}{|c|}{ DAP en $\mathrm{cm}$} \\
\hline & & Roble & Raulí & Coigüe & P. Oregón & Promedio \\
\hline Ladera asoleada 1 & $\begin{array}{l}\text { Media } \\
\text { Error de la media }\end{array}$ & $\begin{aligned} & 9,8 \\
\pm & 0,46\end{aligned}$ & $\begin{array}{c}7,4 \\
\pm 0,50\end{array}$ & $\begin{array}{c}10,3 \\
\pm 0,44\end{array}$ & $\begin{aligned} & 8,1 \\
\pm & 0,40\end{aligned}$ & $\begin{aligned} & 8,9 \\
\pm & 0,45\end{aligned}$ \\
\hline Ladera asoleada 2 & $\begin{array}{l}\text { Media } \\
\text { Error de la media }\end{array}$ & $\begin{array}{c}10,2 \\
\pm 0,50\end{array}$ & $\begin{aligned} & 7,8 \\
\pm & 0,53\end{aligned}$ & $\begin{array}{c}12,1 \\
\pm 0,65\end{array}$ & $\begin{aligned} & 9,0 \\
\pm & 0,47\end{aligned}$ & $\begin{aligned} & 9,7 \\
\pm & 0,54\end{aligned}$ \\
\hline Ladera de sombrío 1 & $\begin{array}{l}\text { Media } \\
\text { Error de la media }\end{array}$ & $\begin{array}{c}10,7 \\
\pm 0,57\end{array}$ & $\begin{array}{c}9,7 \\
\pm 0,44\end{array}$ & $\begin{array}{c}10,9 \\
\pm 0,57\end{array}$ & $\begin{aligned} & 9,5 \\
\pm & 0,44\end{aligned}$ & $\begin{array}{c}10,2 \\
\pm 0,51\end{array}$ \\
\hline Ladera de sombrío 2 & $\begin{array}{l}\text { Media } \\
\text { Error de la media }\end{array}$ & $\begin{array}{c}10,3 \\
\pm 0,57\end{array}$ & $\begin{array}{c}10,3 \\
\pm 0,50\end{array}$ & $\begin{array}{c}10,1 \\
\pm 0,57\end{array}$ & $\begin{array}{c}8,2 \\
\pm 0,37\end{array}$ & $\begin{aligned} & 9,7 \\
\pm & 0,50\end{aligned}$ \\
\hline
\end{tabular}




\section{CUADRO 5}

Promedios en altura (m) y desviaciones estándar por especie en las plantaciones mixtas. Mean values of height measurements and their errors for each tree species in the mixed plantations.

\begin{tabular}{|c|c|c|c|c|c|c|}
\hline & & \multicolumn{5}{|c|}{ Altura en $\mathrm{m}$} \\
\hline & & Roble & Raulí & Coigüe & P. Oregón & Promedio \\
\hline \multirow[t]{2}{*}{ Ladera asoleada 1} & Media & 8,85 & 7,62 & 7,26 & 6,04 & 7,44 \\
\hline & Error de la media & $\pm 0,22$ & $\pm 0,31$ & $\pm 0,21$ & $\pm 0,19$ & $\pm 0,23$ \\
\hline \multirow[t]{2}{*}{ Ladera asoleada 2} & Media & 8,37 & 8,21 & 8,34 & 6,45 & 7,84 \\
\hline & Error de la media & $\pm 0,17$ & $\pm 0,30$ & $\pm 0,24$ & $\pm 0,22$ & $\pm 0,23$ \\
\hline \multirow[t]{2}{*}{ Ladera de sombrío 1} & Media & 8,25 & 8,90 & 7,83 & 6,74 & 7,93 \\
\hline & Error de la media & $\pm 0,27$ & $\pm 0,20$ & $\pm 0,25$ & $\pm 0,18$ & $\pm 0,23$ \\
\hline \multirow[t]{2}{*}{ Ladera de sombrío 2} & Media & 8,06 & 9,10 & 7,39 & 6,13 & 7,67 \\
\hline & Error de la media & $\pm 0,25$ & $\pm 0,27$ & $\pm 0,27$ & $\pm 0,16$ & $\pm 0,24$ \\
\hline
\end{tabular}

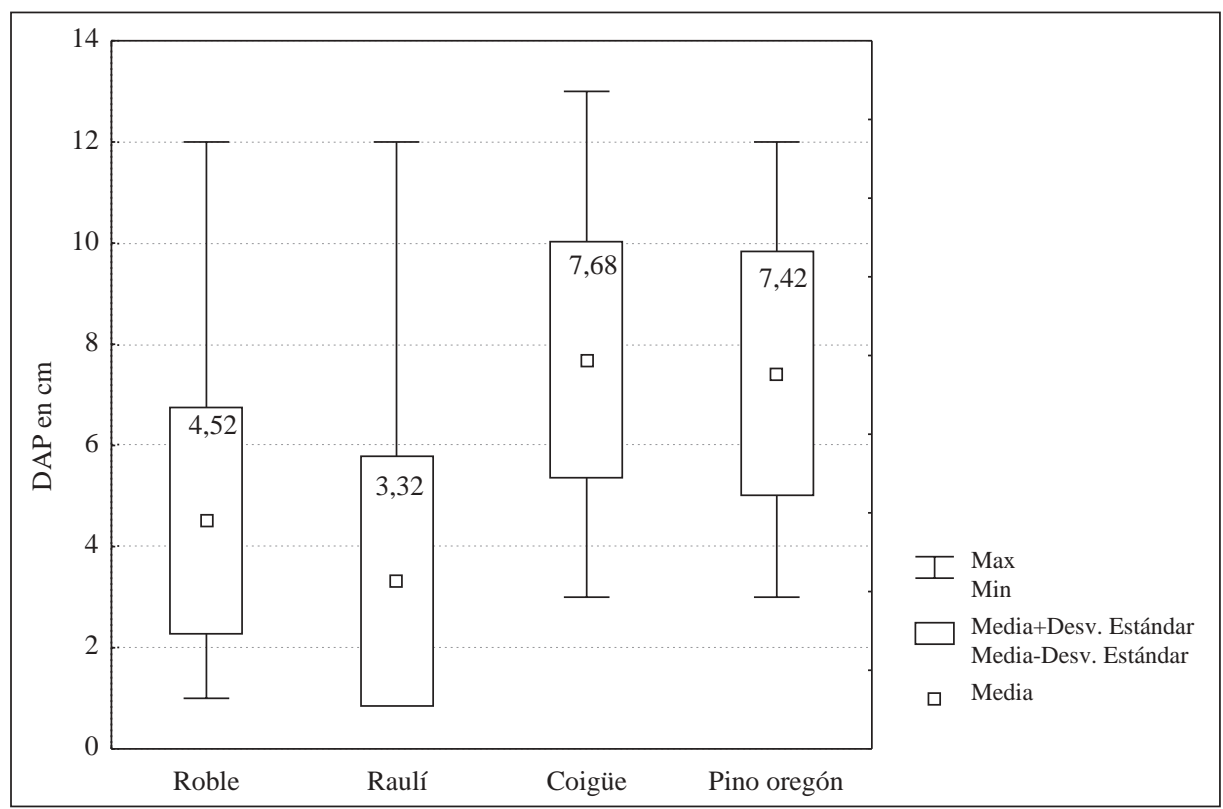

Figura 2. Resultados de los diámetros (DAP) por especie en los claros representados en box plots con sus respectivas medias, desviaciones estándar, valores máximos y mínimos.

Results of diameter measurements for each tree species in the gaps, presented in box plots containing means as well as maximum and minimum values.

El análisis de varianza demuestra diferencia significativa en el factor especie. Sólo entre coigüe y pino oregón estas diferencias son insignificantes, como lo detecta el test de Scheffé, mientras que las diferencias entre las demás especies sí son significativas.

Una influencia significativa de la exposición se da en las especies raulí y pino oregón. Sin embar- 
BOSQUE 24(3): 17-30, 2003

Competencia entre tres especies de Nothofagus y Pseudotsuga menziesii en plantaciones mixtas jóvenes...

go, raulí reaccionó de manera negativa al verse expuesto hacia las laderas asoleadas mientras que el pino oregón mostró una reacción positiva al mismo fenómeno. En total no se encontró una influencia significativa de la exposición sobre el conjunto de las especies en el análisis de varianza.

Los resultados en el desarrollo en altura de las especies en los claros se presentan en el cuadro 6. Raulí tuvo un crecimiento en altura significativamente inferior que las otras especies. Las restantes especies no tuvieron diferencias significativas entre ellas.

La influencia del factor exposición es significativa al considerar el promedio de todas las especies. Sin embargo, el análisis posterior arrojó que eso se debe únicamente al desarrollo inferior del raulí en las laderas asoleadas, las demás especies no difieren significativamente.

Comparando los diámetros y alturas alcanzados en los claros con los de las plantaciones en campo abierto se muestra un desarrollo mucho más lento en los claros, estando esta inferioridad de las plantas en los claros más marcada de lo que se podría explicar por el hecho que tienen un año menos de edad. Se explica este efecto con el clima luminoso amortiguado por la presencia de sombra lateral que suele regir en claros de áreas reducidas.

\section{ESTUDIO DE COMPETENCIA A ESCALA INDIVIDUAL}

\section{Plantaciones en campo abierto}

a) Laderas asoleadas: La figura 3 muestra las posiciones relativas de las cuatro especies en el caso de la plantación en campo abierto y en las laderas asoleadas. Se ve que el roble muestra un conjunto de columnas que disminuyen en tamaño de izquierda a derecha, mientras que para el pino oregón las columnas correspondientes aumentan de tamaño de la izquierda a la derecha; las demás especies no muestran tendencia marcada alguna. Esto significa que roble tiene altos porcentajes en árboles predominantes y codominantes y bajos en ejemplares dominados por uno a cuatro vecinos. Lo contrario es el caso con pino oregón, que muestra un muy bajo porcentaje de árboles dominantes, pero una alta proporción de dominados. Raulí figura en segundo lugar con árboles predominantes y dominantes y coigüe en tercer lugar.

El Test de Kolmogoroff-Smirnoff arroja que roble tiene ventajas significativas ante sus dos vecinos, coigüe y pino oregón. Asimismo, raulí muestra ventajas ante su vecino pino oregón y sólo una tendencia de superioridad con respecto a su vecino coigüe, que no se podía comprobar al nivel de probabilidad de 95\%. La comparación entre roble y raulí no fue posible al no encontrarse estas especies en vecindad directa. Sin embargo, a esta edad, en que la interacción de copas no estuvo muy marcada, se concluye por analogía que si el roble tiene muchas ventajas sobre el coigüe, y el raulí, cuando mucho, leves, este último debe figurar en segundo lugar de una secuencia de competitividad, de la siguiente manera: roble>raulí>coigüe $>$ pino oregón.

\section{CUADRO 6}

Alturas en valores promedios con sus respetivos errores. Means of height measurements and their errors.

\begin{tabular}{|c|c|c|c|c|c|}
\hline & & \multicolumn{4}{|c|}{ Altura en $\mathrm{m}$} \\
\hline & & Roble & Raulí & Coigüe & P. Oregón \\
\hline Claros laderas asoleadas & $\begin{array}{l}\text { Media } \\
\text { Error de la media }\end{array}$ & $\begin{array}{r}4,96 \\
\pm 0,17\end{array}$ & $\begin{array}{c}3,6 \\
\pm 0,18\end{array}$ & $\begin{array}{r}6,34 \\
\pm 0,24\end{array}$ & $\begin{array}{r}6,01 \\
\pm 0,17\end{array}$ \\
\hline Claros laderas de sombrío & $\begin{array}{l}\text { Media } \\
\text { Error de la media }\end{array}$ & $\begin{array}{r}6,03 \\
\pm 0,32\end{array}$ & $\begin{array}{r}5,28 \\
\pm 0,33\end{array}$ & $\begin{array}{r}5,71 \\
\pm 0,24\end{array}$ & $\begin{array}{r}5,30 \\
\pm 0,20\end{array}$ \\
\hline
\end{tabular}


b) Laderas sombrías: La figura 4 muestra una imagen un poco diferente; en exposición sur roble y raulí son altamente dominantes con respecto a sus vecinos, mientras que coigüe tiene pocos árboles y pino oregón aún menos individuos en estas clases, pero sí un muy alto porcentaje en la categoría dominada. Hay diferencia significativa entre estos grupos, pero no entre roble y raulí. La posición relativa del pino oregón en pendiente sur es aún más débil que en la pendiente norte.

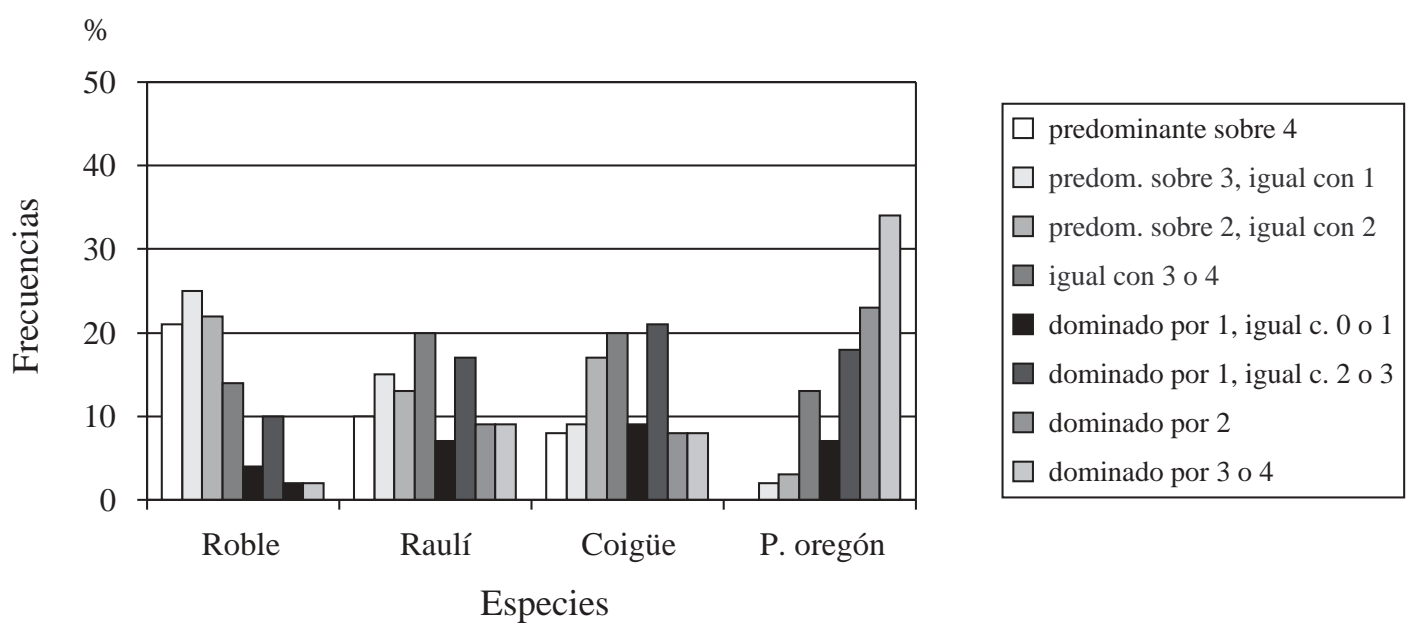

Figura 3. Posición relativa de las especies frente a sus vecinos en las parcelas de las laderas asoleadas en campo abierto. (Las columnas con signaturas diferentes indican el número de vecinos sobre los cuales un individuo domina o bajo los cuales es dominado).

Relative position of the trees of each species with respect to their neighbors in the plots established in the open or on sun exposed slopes.

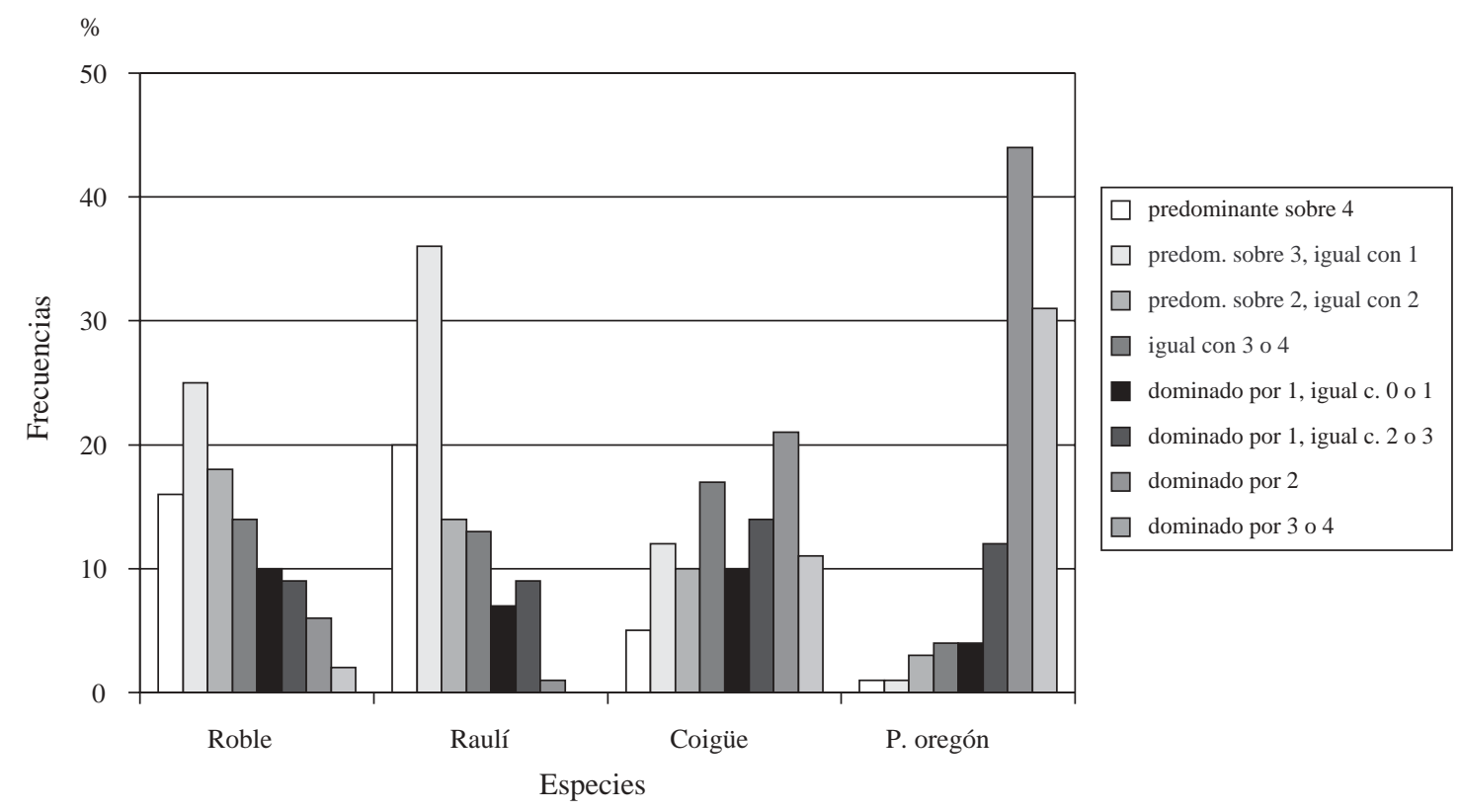

Figura 4. Posición relativa de las especies frente a sus vecinos en las parcelas de campo abierto en las laderas de sombrío. (Las columnas con signaturas diferentes indican el número de vecinos sobre los cuales un individuo domina o bajo los cuales es dominado).

Relative position of the trees of each species with respect to their neighbors in the plots established in the open or on shady slopes. 


\section{SITUACION EN LOS CLAROS}

a) Laderas asoleadas: La posición relativa cambia sustancialmente al pasar del campo abierto hacia los claros. La figura 5 muestra muy altas ventajas de coigüe en los claros en las laderas asoleadas y también un alto comportamiento competitivo de pino oregón. Raulí y roble tienen un bajo nivel de dominancia en estas condiciones. Estas diferencias son significativas entre coigüe y sus especies vecinas. Pino oregón no es vecino directo del coigüe, por lo tanto no lo amenaza la dominancia relativa de este último. Los dos vecinos directos de pino oregón, que pertenecen a las especies roble y raulí, no le ponen problemas de dominancia en esta situación.

b) Laderas sombrías: Los claros en las pendientes sombrías no favorecen tanto al pino oregón como las laderas opuestas, mientras que el roble parece encontrar condiciones marcadamente más favorables: tiene $22 \%$ de árboles predominantes y $14 \%$ que son más altos que tres de sus cuatro vecinos e igual con el cuarto (figura 6). Coigüe también es muy vigoroso en estas condiciones y raulí ocupa la posición más débil. El test de Kolgomoroff-Smirnoff revela que las diferencias entre roble y sus dos vecinos coigüe y pino oregón no son significativas. Lo contrario vale para el raulí que en número de árboles dominantes es significativamente inferior a sus dos vecinos coigüe y pino oregón.

Resumiendo, se puede decir que en la sombra lateral que rige en los claros las especies coigüe y pino oregón tienen más posibilidades que en campo abierto, mientras que el raulí es poco competente en ambas exposiciones. El roble, que en campo abierto ha sido de muy buen comportamiento competitivo en ambas laderas, no muestra el mismo vigor relativo en los claros de las laderas de sombrío.

El cuadro 7 proporciona el número absoluto de árboles/ha que no tienen ningún vecino que sea superior en altura, tanto en el campo abierto como en los claros y en ambas exposiciones.

Evaluación cualitativa: El resultado de la evaluación cualitativa también se muestra en el cuadro 7 , donde bajo la categoría de "buenos" figura el número y porcentaje de los "árboles del futuro". El cuadro muestra también que muchos de los árboles de buena posición en el ranking competitivo tienen defectos cualitativos que los hacen descender a la categoría de "malos", los que deben eliminarse en futuros raleos. Sobre todas las especies y exposiciones estos "candidatos malos" ocupan un $65 \%$.

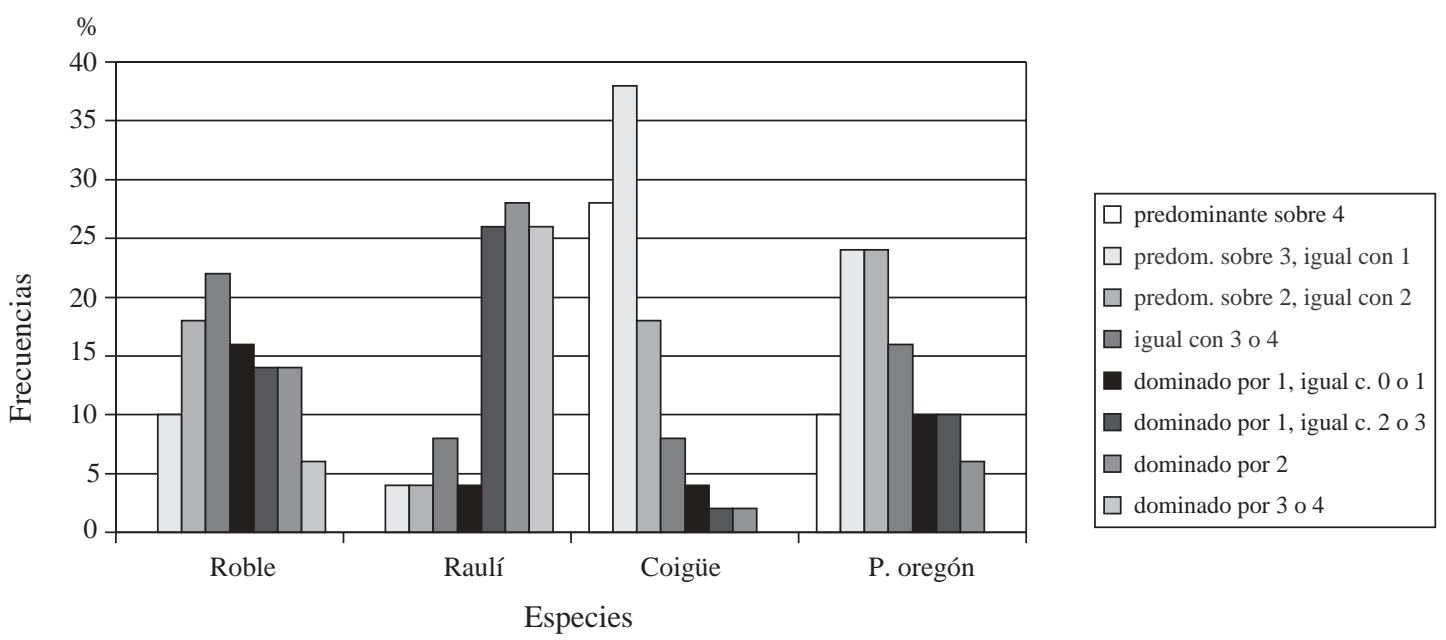

Figura 5. Posición relativa de las especies frente a sus vecinos en los claros en las laderas asoleadas. (Las columnas con signaturas diferentes indican el número de vecinos sobre los cuales domina un individuo o bajo los cuales es dominado)

Relative position of the trees of each species with respect to their neighbors in the gaps on sun exposed slopes. 


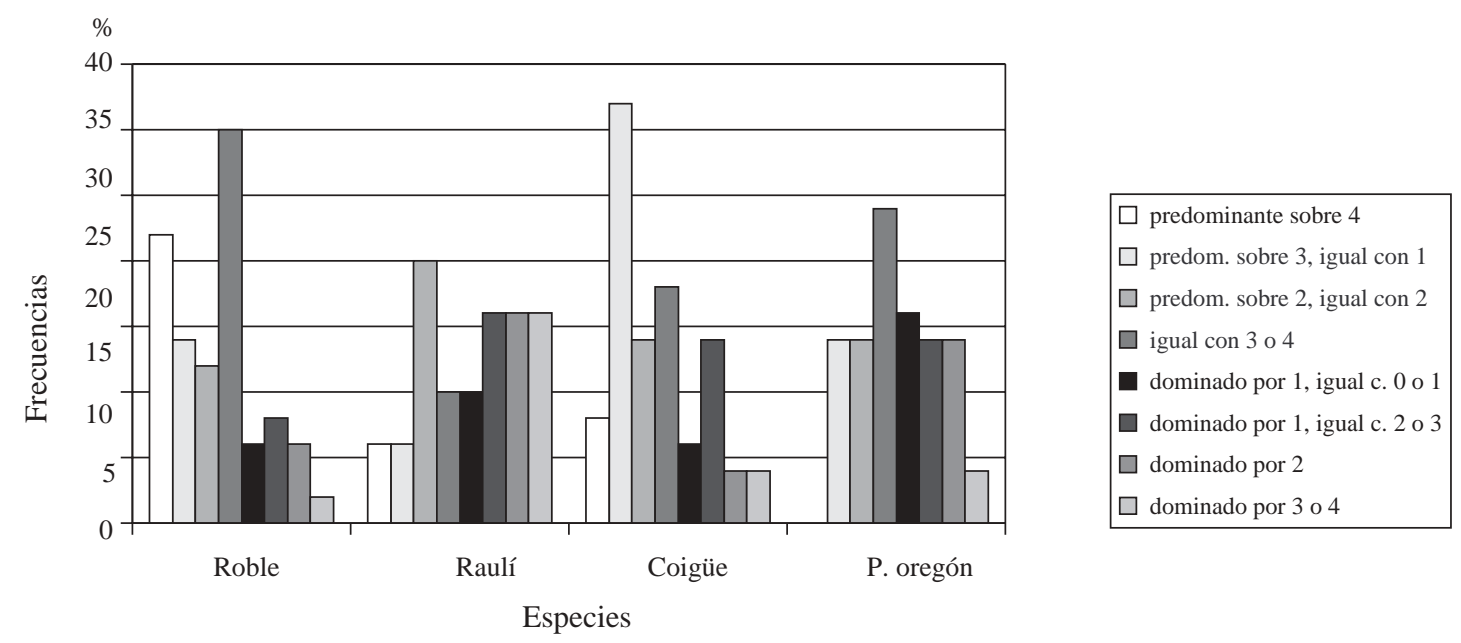

Figura 6. Posición relativa de las especies frente a sus vecinos en los claros de las laderas de sombrío. (Las columnas con signaturas diferenten indican el número de vecinos sobre los cuales un individuo domina o bajo los cuales es dominado).

Relative position of the trees of each species with respect to their neighbors in the gaps on shady slopes.

\section{CUADRO 7}

Número de árboles con buena posición relativa calificados según evaluación fustal en "malos" y "buenos" para las cuatro especies estudiadas.

Number of the trees of each species with a good relative position and denoted in the stem evaluation process as "bad" or "fine" stems.

\begin{tabular}{|c|c|c|c|c|c|c|c|c|c|c|c|c|c|c|c|c|}
\hline \multirow{3}{*}{ Parcela } & \multicolumn{4}{|c|}{ Roble } & \multicolumn{4}{|c|}{ Raulí } & \multicolumn{4}{|c|}{ Coigüe } & \multicolumn{4}{|c|}{ Pino Oregón } \\
\hline & \multicolumn{4}{|c|}{ Número árb. dominantes } & \multicolumn{4}{|c|}{ Número árb. dominantes } & \multicolumn{4}{|c|}{ Número árb. dominantes } & \multicolumn{4}{|c|}{ Número árb. dominantes } \\
\hline & Total & Malos & Buenos & $\%$ & Total & Malos & Buenos & $\%$ & Total & Malos & Buenos & $\%$ & Total & Malos & Buenos & $\%$ \\
\hline Campo abierto & & & & & & & & & & & & & & & & \\
\hline Ladera asoleada & 278 & 140 & 138 & 49 & 203 & 105 & 98 & 48 & 189 & 186 & 3 & 2 & 63 & 46 & 17 & 27 \\
\hline Campo abierto & & & & & & & & & & & & & & & & \\
\hline Ladera de sombrío & 256 & 14 & 42 & 16 & 291 & 238 & 53 & 18 & 154 & 130 & 24 & 16 & 32 & 25 & 7 & 22 \\
\hline Promedio campo abierto & 267 & 177 & 90 & 33 & 247 & 172 & 76 & 33 & 172 & 158 & 14 & 9 & 48 & 46 & 12 & 25 \\
\hline Claros, laderas & & & & & & & & & & & & & & & & \\
\hline Asoleadas & 175 & 63 & 112 & 64 & 56 & 28 & 28 & 50 & 322 & 217 & 105 & 33 & 259 & 126 & 133 & 51 \\
\hline Claros, laderas & & & & & & & & & & & & & & & & \\
\hline De sombrío & 273 & 161 & 112 & 41 & 147 & 77 & 70 & 48 & 252 & 189 & 63 & 25 & 182 & 91 & 91 & $\mathbf{5 0}$ \\
\hline Promedios claros & 224 & 112 & 112 & 53 & 102 & 53 & 49 & 49 & 287 & 203 & 84 & 29 & 221 & 109 & 112 & 51 \\
\hline
\end{tabular}


BOSQUE 24(3): 17-30, 2003

Competencia entre tres especies de Nothofagus y Pseudotsuga menziesii en plantaciones mixtas jóvenes...

Además se desprende de las cifras que el porcentaje de árboles de buena calidad siempre es mayor en los claros que en plantaciones hechas en campo abierto y -con la excepción de coigüe en campo abierto, donde esta especie sufre mucho de daños de insolación-, la tasa de árboles del futuro es generalmente más alta en las laderas asoleadas que en las laderas de sombrío. Cabe mencionar, también, que el pino oregón hasta la fecha tiene muy pocos árboles del futuro en las plantaciones en campo abierto, y sólo es prometedor como especie en mezcla con las demás en los claros. Calculado sobre todas las condiciones de sitio, tienen mayor porcentaje de "árboles de buena calidad" roble y raulí, seguido por pino oregón, mientras que el coigüe ocupa el último lugar.

\section{DISCUSION}

Si bien es cierto que se ha trabajado en Chile ya desde mucho tiempo sobre crecimiento de rodales mixtos de roble, raulí y coigüe (Rocuant 1974, Grosse 1987, Donoso et al. 1993, entre otros), esta información se refiere en la mayoría de los casos a renovales siendo más escasas las informaciones sobre plantaciones. Para comparar los crecimientos en diámetro y altura de las plantaciones mixtas en Arquilhue puede recurrirse al trabajo de Donoso et al., en el que se informa sobre parámetros de crecimiento en 18 plantaciones entre 10 y 18 años de edad. Estos autores concluyen que los crecimientos de los primeros 10 años son mejor en coigüe en comparación con roble y raulí, hecho que se comprobó en este estudio sólo para las laderas asoleadas, mientras que en las sombrías hay prácticamente igualdad en los DAP entre las tres especies. Refiriéndose al raulí, en especial, éste obviamente no se encuentra en su óptimo en las plantaciones estudiadas de Arquilhue, quedándose más de 1,6 cm atrás de las indicaciones de Donoso para el caso de Rucatayo (DAP promedio 7,6 cm contra 9,2 cm) en la misma exposición norte. Es posible que esta inferioridad se deba a la baja altitud s.n.m. en que se encuentran las plantaciones de estudio en Arquilhue, la que con 200 ms.n.m. está más abajo del rango natural de la especie raulí, y donde las laderas con mucho sol se calientan más que a los $850 \mathrm{~m}$ de Rucatayo. En la ladera sombría, sin embargo, no tiene este inconveniente y supera al raulí de Rucatayo en crecimiento de diámetro y altura.

No se han encontrado casos comparables en la literatura chilena que incluyan pino oregón. En Europa se ha trabajado mucho sobre mezclas entre Pseudotsuga menziesii y Fagus silvatica (Otto 1987, Burschel und Huss 1997), llegando a la conclusión de que el Haya como especie muy tolerante es la única especie europea que se puede manejar junto con el pino oregón por aguantar -en un estrato inferior- la competencia que ejerce este último. Cualquier otra mezcla con Pseudotsuga está condenada a un fracaso por la superioridad excesiva en crecimiento que posee el pino oregón en aquellos latitudes.

Esta experiencia no se puede hacer extensiva al caso chileno, como muestran los resultados elaborados en este trabajo.

\section{CONCLUSIONES}

El presente estudio de competencia a escala individual, en combinación con la evaluación cualitativa, permiten sacar algunas conclusiones para un primer raleo de las plantaciones efectuadas en campo abierto. En los claros se está todavía lejos de este momento de manera que quedan 6 años más para observar la dinámica del desarrollo en altura y calidad.

Los raleos pueden perseguir diferentes objetivos, como, por ejemplo, bajar la densidad, garantizar porcentajes de mezcla y/o maximizar el incremento en valor, liberando los "arboles del futuro" de competidores indeseados y así concentrando el incremento en estos candidatos preseleccionados. En el presente caso no es necesario todavía bajar la densidad en forma general. El espaciamiento inicial ha sido $2,5 \times 2,5 \mathrm{~m}$, la cuota de mortalidad ha sido alrededor de 200 árboles por ha, por lo cual se cuenta hoy con alrededor de 1.400 árboles por ha, lo que dada la altura de alrededor de $10 \mathrm{~m}$ no ha conducido a roces entre las copas todavía. Tampoco hay necesidad de intervenir para garantizar porcentajes de mezcla ya que hasta ahora la proporción de $25 \%$ que mantiene cada una de las especies desde la edad de su plantación no ha cambiado sustancialmente.

Con la optimización del desarrollo en valor la situación es distinta: De los árboles con buena posición en el proceso competitivo hay un alto 
porcentaje de individuos de mala calidad, los que pueden impedir el desarrollo de un número suficiente de árboles, prometedores en posición relativa y valor fustal, que se necesita para maximizar la producción en términos de valor. Este número puede derivarse del área basal meta que se quiere tener, efectuado el último raleo. De un trabajo de Puente et al. (1981) se desprende un valor de 41 $\mathrm{m}^{2} /$ ha como promedio de un rango que estos autores proporcionan como áreas basales meta en renovales de roble/raulí. Anhelando un DAP meta de $60 \mathrm{~cm}$ (Avilés 1993, Pokorny 1995) y una reserva de un $30 \%$ para compensar futuras pérdidas de árboles del futuro, se llega a un número meta de 190 árboles. En el cuadro 7 se puede apreciar que hay sitios dentro de los latizales mixtos que no cumplen estas condiciones numéricas, como p.e. los de las laderas de sombrío donde hay sólo 126 buenos candidatos, entre ellos algunos que tienen un competidor de igual altura y mala calidad. Es por ello que se aconseja la realización pronta de un primer raleo aunque sea precomercial. Esta intervención tiene que ser un raleo por lo alto, concentrándose en los árboles de buena posición relativa pero de mala calidad.

Para completar el número deficiente de buenos candidatos en las laderas de sombrío al nivel de 190 árboles, es necesario recurrir al colectivo de árboles que tienen más de un competidor codominante y liberarlos tan enérgicamente que no tengan obstáculo en emerger en el lapso hasta el segundo raleo. Determinando como "árboles a eliminar" todos aquellos que son de mala calidad, predominantes o codominantes con por lo menos un competidor de buena calidad se llega a una intensidad de la intervención que en las laderas asoleadas se eleva a alrededor de 200 árboles y 350 en laderas de sombrío. El cuadro 8 informa cómo se reparte esta intervención sobre las cuatro especies. En términos de área basal esto corresponde a $2,3 \mathrm{~m}^{2}(=24 \%)$ por ha en las laderas asoleadas y $4 \mathrm{~m}^{2}(=36 \%)$ por ha en las laderas de sombrío, si se calcula con un DAP de $12 \mathrm{~cm}$ del árbol eliminado en promedio.

Cabe señalar que la recomendación de efectuar el primer raleo entre 11 y 13 años de edad en los rodales mixtos estudiados se encuentra en buena concordancia con lo propuesto por Donoso et al. (1993), quienes indican el lapso entre la culminación del crecimiento anual corriente y el momento en que el crecimiento medio empieza a bajar, es decir, entre 10 y 15 años como el tiempo más adecuado. Estas recomendaciones se orientan al concepto de una máxima respuesta de los árboles remanentes después del raleo. En el caso de rodales mixtos con especies de distinto ritmo de crecimiento y con metas de producir madera de alto valor hay un argumento más que hace obligatorio una edad tan joven para la primera intervención: llegar a tiempo para mantener la mezcla y no perder fustes de calidad en la fuerte competencia interespecífica.

\section{AGRADECIMIENTOS}

Este artículo se basa en una tesis de magíster entregada en la Facultad de Ciencias Forestales y Ecología de los Bosques de la Universidad de Göttingen, Alemania, y contó con la colaboración

\section{CUADRO 8}

Número y porcentaje de árboles de cada especie a eliminar por ha en la primera intervención. Number and percentage of trees of each species to be eliminated during the first thinning operation.

\begin{tabular}{|c|c|c|c|c|c|c|c|c|}
\hline \multirow{2}{*}{ Parcela } & \multicolumn{2}{|c|}{ Roble } & \multicolumn{2}{|c|}{ Raulí } & \multicolumn{2}{c|}{ Coigüe } & \multicolumn{2}{c|}{ Pino Oregón } \\
\cline { 2 - 9 } & Número & $\%$ & Número & $\%$ & Número & $\%$ & Número & $\%$ \\
\hline Campo abierto, laderas asoleadas & 81 & 41 & 50 & 25 & 60 & 31 & 7 & 3 \\
\hline Campo abierto, laderas de sombrío & 126 & 36 & 169 & 48 & 49 & 14 & 8 & 2 \\
\hline
\end{tabular}


BOSQUE 24(3): 17-30, 2003

Competencia entre tres especies de Nothofagus y Pseudotsuga menziesii en plantaciones mixtas jóvenes...

de la Empresa Agrícola y Forestal Taquihue en su fundo Arquilhue. Se le agradecen a los responsables de esta empresa las facilidades prestadas para un buen desarrollo de esta investigación.

\section{BIBLIOGRAFIA}

AVILES, B. 1993. Untersuchungen zur waldbaulichen Behandlung und Bewirtschaftung von Renovalesbestände in Mittelchile. (Estudio sobre el tratamiento silvícola y el manejo de renovales en el Centro-Sur de Chile). Trabajo doctoral, Facultad de Ciencias Forestales, Universidad de Freiburg, Alemania. $174 \mathrm{p}$

BURSCHEL, P. und HUSS, J. 1997. Grundriss des Waldbaus - ein Leitfaden fuer Studium und Praxis Paul Parey. Hamburg und Berlin. 487 pp.

GROSSE, H. 1987. "Desarrollo de Renovales de Raulí raleados", Ciencias e Investigación Vol. 1 (2): 31-43.

GROSSE, H. 1987. "Desarrollo inicial de plantaciones de Raulî", Ciencias e Investigación Vol. 1 (1): 49-56.

DONOSO, P., C. MONFIL, L. OTERO, L. BARRAIES. 1993.

"Estudio de crecimiento de plantaciones y renovales manejados de especies nativas en el área andina de las provin- cias de Cautin y Valdivia", Ciencia e Investigación Forestal 7 (2): 253-287.

OTTO, H.J. 1987. "Skizze eines optimalen Douglaseinwaldbaus in Nordwestdeutschland FoHo 42", Jahrg: 515-522.

POKORNY, B. 1995. Zur Überführung von mittelchilenischen Nothofagus-Renovalesbeständen in Wirtschaftswälder (Aportes para la conversión de Renovales del género Nothofagus en bosque manejado en el Sur de Chile). Diss. Forstl. Fak. der Univ. Freiburg, Alemania. 199 p.

PUENTE, M., R. PEÑALOZA, C. DONOSO, R. PAREDES, P. NUÑEZ, R. MORALES, O. ENGDAHL. 1981. Estudio de raleo y otras técnicas para el manejo de renovales de Raulí y Roble. Instalación de ensayos de raleo. Investigación y Desarrollo Forestal: Docto. de Trabajo No 41 . FO: DP/CHI/ 76 / 003. Santiago de Chile. 74 p.

ROCUANT, L. 1974. "Raleos en Renovales de Roble-Raulî", Actas del Primer Seminario "Situación actual y posibilidades futuras del Manejo de los Renovales en Chile". CONAF / 1974: 19-40.

SIEBERT, H. 1999. La silvicultura alternativa: Un concepto silvícola para el bosque nativo chileno. En: Silvicultura de los bosques nativos de Chile. Ed. Universitaria, Santiago, pp. 381-406.

SACHS, L. 1984. Angewandte Statistik (Estadística aplicada) 6. Auflage Springer-Verlag Berlin, Heidelberg, New York, Tokyo, $552 \mathrm{p}$.

YANDELL, B. 1997. Practical data analysis for designed experiments.1. Ed. Chapman \& London. 437 p. 